\title{
Perfil epidemiológico dos casos de dengue no estado de Pernambuco, Brasil
}

\author{
Epidemiological profile of dengue cases in the state of Pernambuco, Brazil \\ Perfil epidemiológico de los casos de dengue en el estado de Pernambuco, Brasil
}

\author{
Carlos Antonio de Lima Filho \\ ORCID: https://orcid.org/0000-0001-5517-0347 \\ Universidade Federal de Pernambuco, Brasil \\ E-mail: cttoni2000@gmail.com \\ Anderson Emanuel da Silva Lima \\ ORCID: https://orcid.org/0000-0002-9455-9045 \\ Universidade Federal de Pernambuco, Brasil \\ E-mail: Anderson.55silva@hotmail.com \\ Rúbia Maria Golberto Arcanjo \\ ORCID: https://orcid.org/0000-0002-8038-8791 \\ Centro Universitário Facol, Brasil \\ E-mail: rubiaarcanjo123@gmail.com \\ Daniela de Lira Silva \\ ORCID: https://orcid.org/0000-0001-8145-2508 \\ Universidade Federal de Pernambuco, Brasil \\ E-mail: daniela.lirasilva@ufpe.br \\ Geovanna Figueirêdo de Jesus \\ ORCID: https://orcid.org/0000-0002-2485-5279 \\ Universidade Federal de Pernambuco, Brasil \\ E-mail: geovannafj29@icloud.com \\ Amanda Oliveira Bernardino Cavalcanti de Albuquerque \\ ORCID: https://orcid.org/0000-0002-1011-8964 \\ Universidade de Pernambuco, Brasil \\ E-mail: amandaobernardino@hotmail.com \\ Aline Papin Roedas da Silva \\ ORCID: https://orcid.org/0000-0002-6511-3407 \\ Prefeitura Municipal de Bauru, Brasil \\ E-mail: aline.papin@gmail.com \\ Matheus Vinicius Barbosa da Silva \\ ORCID: https://orcid.org/0000-0003-1295-6301 \\ Universidade Federal de Pernambuco, Brasil \\ E-mail: matheushue30@gmail.com
}

\begin{abstract}
Resumo
Objetivo: avaliar o perfil epidemiológico da dengue no estado de Pernambuco no período de 2015 a 2020. Metodologia: Trata-se de um estudo retrospectivo, descritivo com abordagem qualitativa sobre o perfil epidemiológicos dos casos de dengue no estado de Pernambuco no período de 2015 a 2020. A coleta de dados foi realizada no Sistema de Notificação de Agravo de Notificação (SINAN), vinculado ao Departamento de Informática do Sistema Único de Saúde (DATASUS), com a coleta de dados sendo realizada em janeiro de 2022. Resultados: No período estudado foram notificados 252.695 casos de dengue, sendo que a maioria foi no público feminino (59\%), de faixa etária dos 20-39 (36,08\%), de raça parda (40,5\%), com uma alta prevalência dos menos escolarizados. A maioria dos casos foram de dengue clássica diagnosticado por métodos clínicos-epidemiológicos evoluindo para cura, sendo que o sorotipo mais prevalente ainda se apresenta desconhecidos, com maior número nos meses mais quente. Conclusão: Ficou em evidência que a dengue ainda é bem prevalente no estado de Pernambuco, a subnotificação é possíveis erros no momento da notificação é um grande desafio apresentado, o que pode dificultar ações de combate a doença.
\end{abstract}

Palavras-chave: Dengue; Aedes; Epidemiologia; Saúde pública; Monitoramento epidemiológico.

\begin{abstract}
Objective: to evaluate the epidemiological profile of dengue in the state of Pernambuco from 2015 to 2020. Methodology: This is a retrospective, descriptive study with a qualitative approach on the epidemiological profile of dengue cases in the state of Pernambuco from 2015 to 2020. Data collection was carried out in the Notification System for Notifiable Diseases (SINAN), linked to the Department of Informatics of the Unified Health System (DATASUS), with data collection being carried out in January 2022. Results: In the period studied, 252,695 cases of dengue were reported, most of which were female (59\%), aged 20-39 (36.08\%), of mixed race (40.5\%), with a high
\end{abstract}


prevalence of less educated. Most cases were of classic dengue diagnosed by clinical-epidemiological methods evolving to cure, and the most prevalent serotype is still unknown, with a greater number in the warmer months. Conclusion: It was evident that dengue is still very prevalent in the state of Pernambuco, underreporting and possible errors at the time of notification is a great challenge presented, which can make it difficult to combat the disease.

Keywords: Dengue; Aedes; Epidemiology; Public health; Epidemiological monitoring.

\section{Resumen}

Objetivo: evaluar el perfil epidemiológico del dengue en el estado de Pernambuco de 2015 a 2020. Metodología: Se trata de un estudio descriptivo retrospectivo con abordaje cualitativo sobre el perfil epidemiológico de los casos de dengue en el estado de Pernambuco de 2015 a 2020. Datos la recolección se realizó en el Sistema de Notificación de Enfermedades de Declaración Obligatoria (SINAN), vinculado al Departamento de Informática del Sistema Único de Salud (DATASUS), habiéndose realizado la recolección de datos en enero de 2022. Resultados: En el período estudiado se registraron 252.695 casos de dengue fueron notificados, siendo la mayoría del sexo femenino (59\%), de 20 a 39 años $(36,08 \%)$, mestizos $(40,5 \%)$, con alta prevalencia de menor escolaridad. La mayoría de los casos fueron de dengue clásico diagnosticados por métodos clínico-epidemiológicos que evolucionaron a la curación, y aún se desconoce el serotipo más prevalente, con mayor número en los meses cálidos. Conclusión: se evidenció que el dengue todavía es muy prevalente en el estado de Pernambuco, el subregistro y los posibles errores en el momento de la notificación es un gran desafío que se presenta, lo que puede dificultar el combate a la enfermedad.

Palabras clave: Dengue; Aedes; Epidemiología; Salud pública; Monitoreo epidemiológico.

\section{Introdução}

A dengue é uma doença febril ocasionada por um arbovírus, que se divide em quatros tipos diferentes distintos, DENV 1, DENV 2, DENV 3 e DENV 4 (Brasil, 2021). A dengue é transmitida pelos mosquitos do gênero Aedes, sendo o Aedes aegypti o principal vetor, o Aedes albopictus, é considerado atualmente um vetor de transmissão secundária (Braga et al., 2007). O diagnóstico pode ser realizado por critérios clínicos-epidemiológicos e laboratoriais, não possuindo tratamento específico, apenas sintomáticos e hidratação (Dias et al., 2010).

O ciclo de transmissão ocorre quando o principal vetor da doença no Brasil, o Aedes aegypti, pica uma pessoa infectada com um dos sorotipos do vírus, cerca de uma semana depois o vírus migra para a sua glândula salivar, tornando o mosquito propenso a infectar outras pessoas durante a picada (Campos et al., 2018). O Aedes aegypti mede cerca de 1cm, ele possui uma coloração escura com listras brancas na patas, cabeça e corpo, para se reproduzir ele necessita de locais com água parada, no verão, com a combinação de temperaturas mais quentes e chuvas a sua circulação é intensificada (Rio Grande do Sul, 2021). Para Santos et al. (2019) pelo fato do Brasil apresentar um clima predominante tropical, fornece condições ideais para a proliferação do mosquito. Segundo Zara et al. (2016) o primeiro registro do Aedes aegypti foi descrito em 1762, no Egito, no Brasil, acredita que sua introdução se deu no período colonial, entre os séculos XVI e XIX, durante o comércio de escravo.

Relatos sugerem que a circulação do DENV no Brasil ocorre desde o século XIX, porém só foi comprovada em 1982 com o isolamento dos sorotipos DENV 1 e DENV 4, a década de 1980 foi marcada pela disseminação da doença pelos os estados brasileiros e a ocorrência da primeira epidemia, a partir da década de 1990 com a introdução do sorotipo DENV 4 foram relatados os primeiros casos grave da doença (Cavalcanti et al., 2018).

A Organização Mundial da Saúde (OMS) estima que 2,5 bilhões estão sob o risco de contrair a dengue, sendo que anualmente ocorre cerca de 50 milhões de casos. Por ser um problema de saúde pública do Brasil, a portaria $\mathrm{N}^{\mathrm{O}} 204$, de 17 de fevereiro de 2016 trás a dengue com uma das doenças da Lista Nacional de Notificação Compulsória de doenças e agravos (Santos et al., 2019/ Brasil, 2016). Segundo Teixeira et al. (2013) as principais epidemia da dengue ocorrem nas regiões sudeste e nordeste, com a presença de uma grande quantidade de casos e de óbitos. Entre os anos de 2013 a 2020 foram notificados mais de um milhão de casos de na região nordeste e 707 mortes, o que corresponde a 18,1\% e 22,3\% de todo o pais, respectivamente, no mesmo período o estado de Pernambuco foi responsável por 20,1\% dos casos e 19,4\% dos óbitos por dengue na região nordeste (Nascimento et al., 2020). 
Segundo Cordeiro et al. (2008) o primeiro surto da doença em Pernambuco ocorreu em 1987, que prosseguindo 1995 com a ocorrência da epidemia causado pelo DENV 2. Atualmente a doença ainda apresenta uma alta prevalência no estado, no ano de 2021 houve um aumento de 89,4\% em comparação com o ano de 2020 (Brasil, 2021b).

Perante o que foi comentado, é de suma importância a realização de estudos epidemiológicos em regiões que são endêmicas, para tentar compreender os fatores que contribuem para a continuidade da doença, e para auxiliar os gestores e profissionais de saúde sobre medidas para o seu combate. O objetivo desse trabalho e avaliar o perfil epidemiológico da dengue no estado de Pernambuco no período de 2015 a 2020.

\section{Metodologia}

Essa pesquisa trata-se de um estudo retrospectivo, descritivo, com abordagem quantitativa (Hochman et al., 2005). Estudo realizado através de dados epidemiológicos através do Sistema de Informação de Agravos de Notificação (SINAN), que é uma base de dados vinculados ao Departamento de Informática do Sistema Único de Saúde (DATASUS). O SINAN permite uma consolidação continuada dos dados, avaliação e monitoramento das intervenções referente ao controle de doença no país, também serve se suporte para compra de medicamentos e insumos de saúde (Rocha et al., 2020).

O estado de Pernambuco é localizado na região Nordeste do Brasil, fazendo limite com os estados da Paraíba, Ceará, Alagoas, Piauí e Bahia, banhado pelo oceano Atlântico, possuindo cerca de 187 km de litoral. Em 2010 tinha uma população de 8.796.448 habitantes, divididos em 184 municípios mais o arquipélago de Fernando de Noronha, sendo o segundo estado mais populoso do nordeste, e o sétimo do Brasil (IBGE, 2021). Segundo Santo et al. (2012) os municípios do estado são distribuídos entre 11 Gerências Regionais de Saúde, as Geres, sendo que cada uma abrange um certo contorno territorial, as microrregiões de saúde, as Geres tem a função de interlocução entre os municípios e esfera gestora estadual de educação.

A coleta de dados foi realizada através do acesso ao banco de dados do SINAN/DATASUS, a coleta se apoiou no período de 2015 a 2020, as variáveis utilizadas foram números de casos do período, sexo, raça, meses do ano, escolaridade, forma de confirmação, sorotipo, tipo de saída e faixa etária. Na Figura 1 é possível observar como se desenvolveu a coleta de dados da pesquisa. O programa Excel 2010 foi utilizado para o auxilio da análise estatística dos dados. Os dados coletados foram distribuídos em gráficos e tabelas para sua melhor compreensão. Por se tratar de informações de acesso público, o artigo primeiro da resolução número 510, de abril de 2016, do Conselho Nacional de Saúde, descarta a acareação do mesmo pelo comitê de ética em pesquisa (Brasil, 2016).

Figura 1. Procedimentos da coleta de dados.

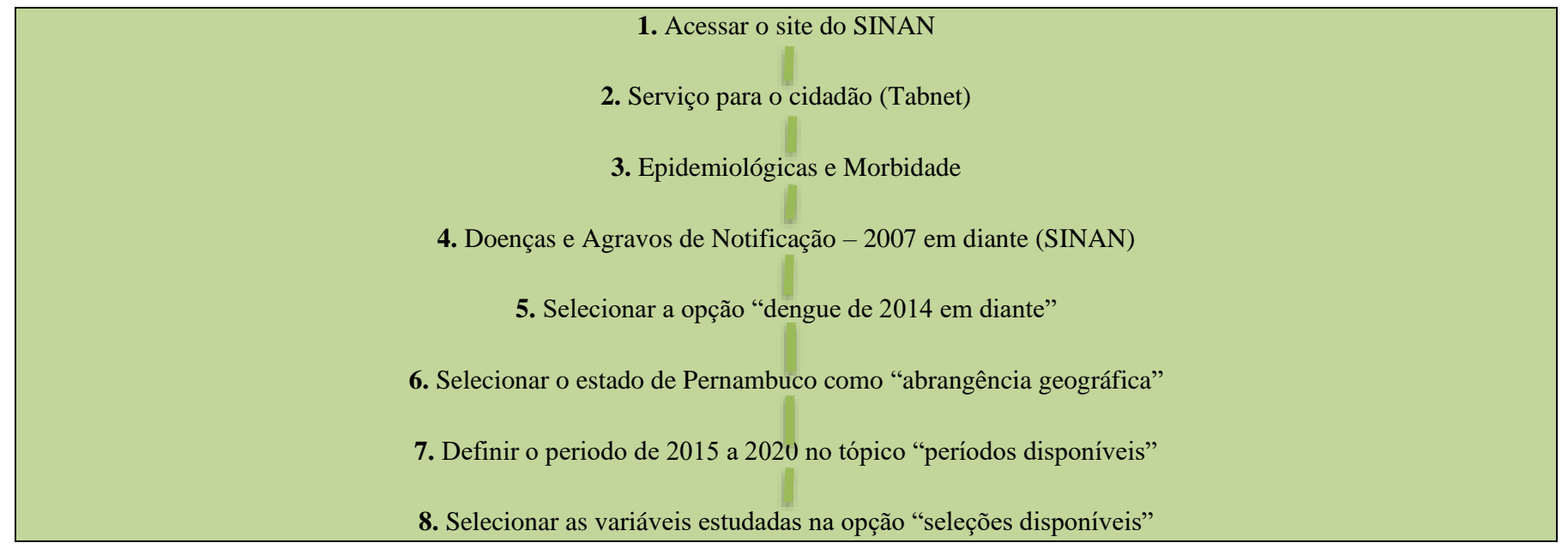

Fonte: Autores (2022). 


\section{Resultados e Discussão}

No período estudado foram notificados 252.695 casos de dengue no estado de Pernambuco. O Gráfico 1 apresenta a distribuição dos casos durante o período de tempo estudado. No Gráfico 1 observa-se que o ano que apresentou o maior número de casos foi o de 2015 (44,0\%), após uma drástica queda o estado alcançou no ano de 2017 o menor número de casos $(3,1 \%)$, posterior a esse período é possível notar que apesar de apresentar baixos números de casos notificados o estado apresenta uma variação de queda e aumento de casos entre os anos de 2018 a 2020.

Gráfico 1. Casos de dengue notificados no estado de Pernambuco no período de 2015 a 2020.

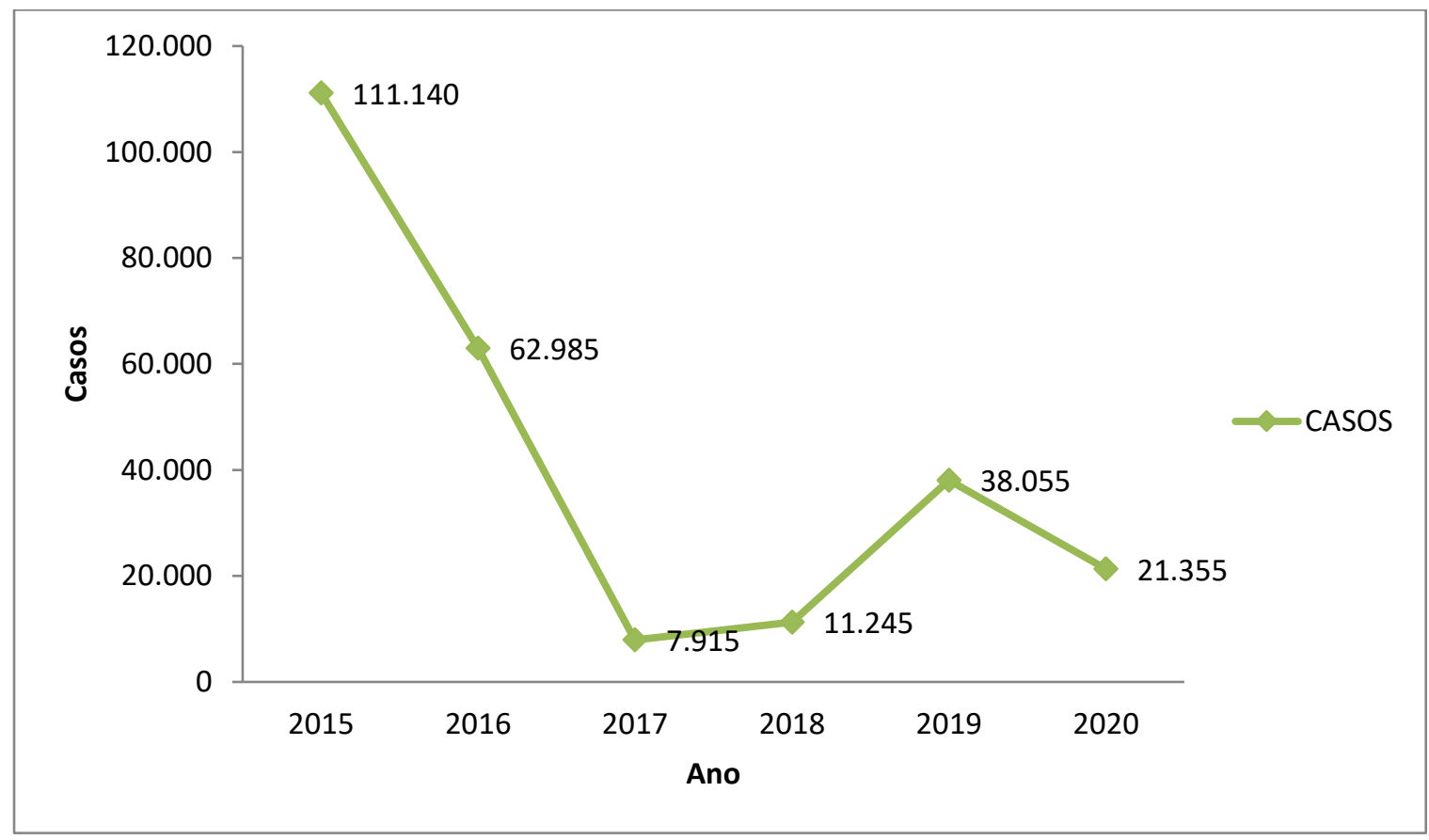

Fonte: Autores (2022). A partir de dados coletados do SINAN/DATASUS.

Observando o Gráfico 1 é possível constatar que a maioria dos casos ocorreram no ano de 2015 (44,0\%) e 2016 $(24,9 \%)$. Esse período foi marcado pela epidemia de dengue que ocorreu no Brasil, sendo que em 2015 foi o ano mais epidêmico do pais, apresentando o maior número de casos e hospitalizações já notificados (Brasil, 2017b). O gráfico mostra que Pernambuco também apresentou uma epidemia de dengue no período, outras localidades também apresentaram uma epidemia de dengue, como a cidade de Pinhalzinho, em Santa Catarina, evidenciado pelo estudo de Andrioli et al. (2020). A queda nos números de casos observado pode ser pelo fato da ocorrência de subnotificação, atraso das notificação e erros no momento da digitação dos dados, fatores que estão interligados com a falta de treinamento dos profissionais envolvido na notificação (Duarte et al., 2006).

Em relação ao sexo, no Gráfico 2 ficou evidente uma maior prevalência do sexo feminino com 59\% dos casos (147.627) em comparação com o masculino, que somaram 41\% (104.701), ainda houve a notificação de 367 casos que foram notificados como ignorado ou branco, que por apresentar menos de $1 \%$ dos casos, foram desconsiderados na elaboração do gráfico. 
Gráfico 2. Casos notificados de dengue segundo o sexo, no estado de Pernambuco, no período de 2015 a 2020.

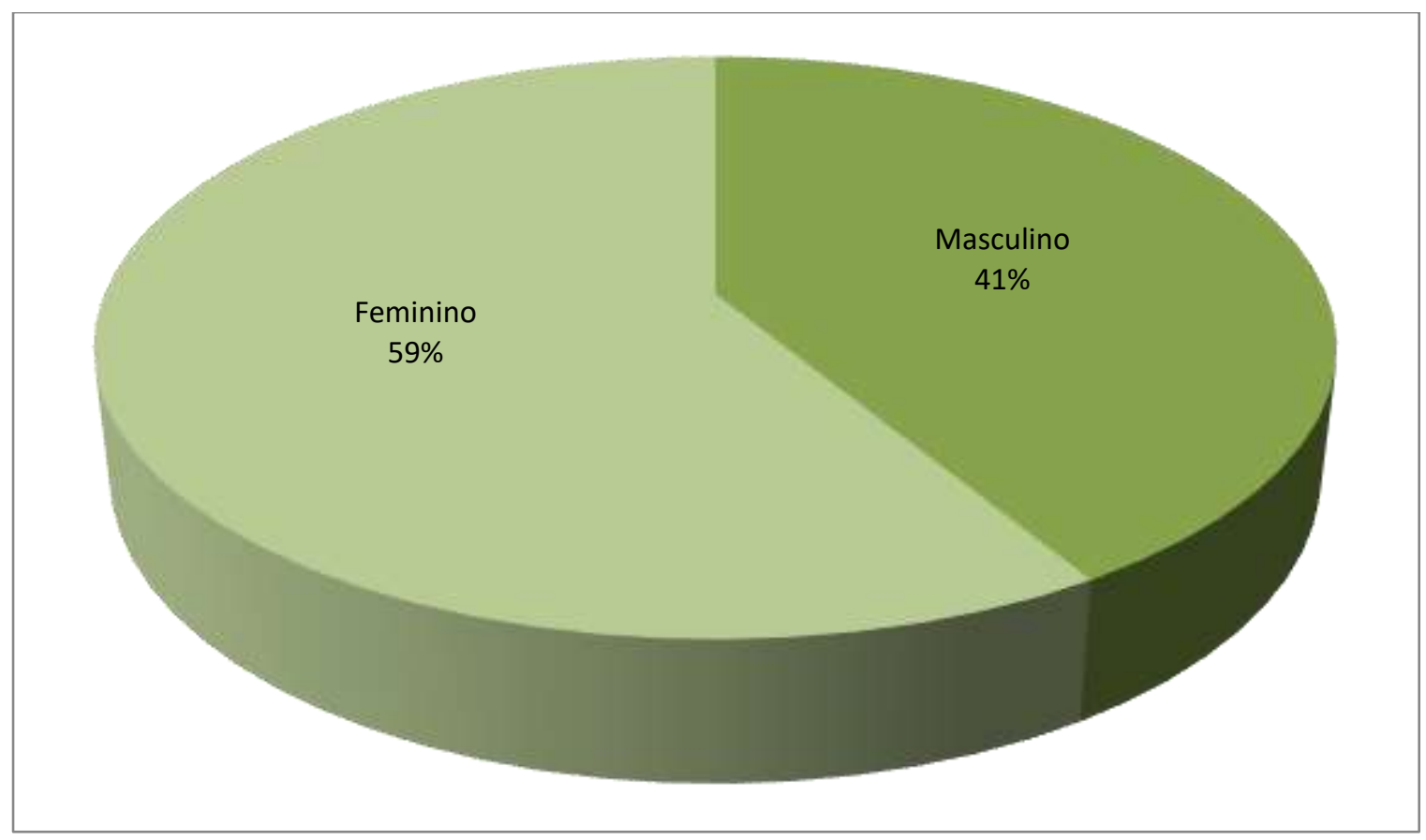

Fonte: Autores (2022). A partir de dados coletados do SINAN/DATASUS.

Através do Gráfico 2, observa-se que a maioria dos casos ocorreu nas mulheres, esse achado vai de encontro com os estudos de Lucena et al. (2019) e de Oliveira et al. (2019) que em seus estudos oberveu as mulheres representaram 53,9\% e $58 \%$ dos casos, respectivamente. Segundo Guedes et al. (2019) a provavel explicação para essa caracteristica esta associado a baixa ocorrência de casos graves da doença, então assim, pelo fato de apresentar sintomas leves da doença os homens não procura assistencia a saúde, consequentemente o caso não é notificado. Outra possível explicação para a maior ocorrência de casos em mulheres e o fato do Aedes aegypti esta em maior circulação em locais intradomiciliar e peridomiciliar, locais comumente é mais frequentado por mulheres (Scandar et al., 2010).

O Gráfico 3 a relação da dengue com a raça, sendo que apresentou a maioria dos casos foi a raça parda com 102.453 (40,54\%), porém é possível observar que os casos que se encontram como ignorado/branco se apresenta em um patamar avançado $(46,47 \%)$. 
Gráfico 3. Casos notificados de dengue segundo raça, no estado de Pernambuco, no período de 2015 a 2020.

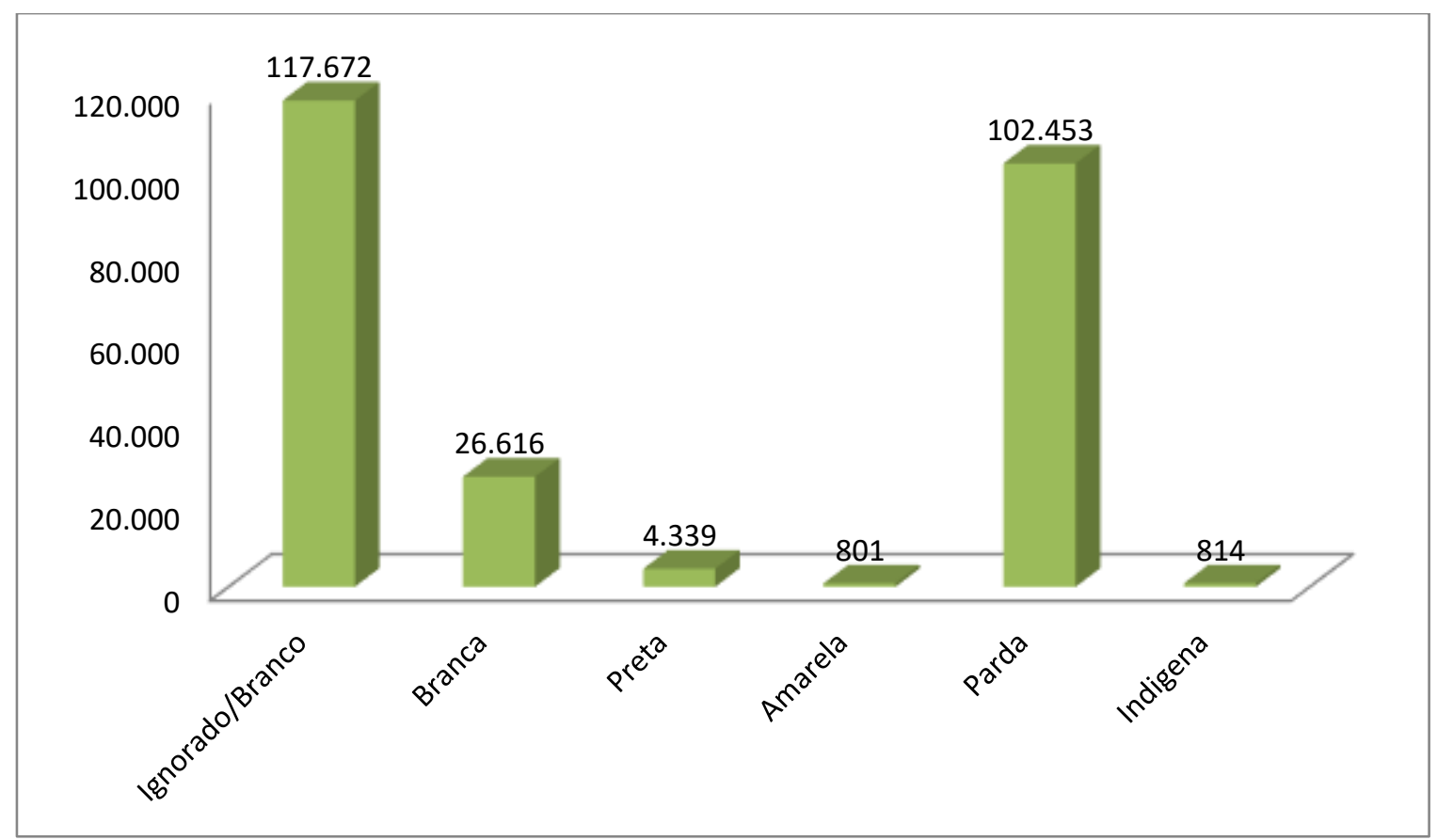

Fonte: Autores (2022). A partir de dados coletados do SINAN/DATASUS.

Os achados do Gráfico 3 mostram que a maioria dos casos foram notificados como ignorado/branco (46,6\%), uma das possiveis causas para esse fato poder ser explicado por um erro no momento da notificação, contudo analisarmos que a raça com maior número de casos são em pessoas pardas, apresentando 40,5\% dos casos, esses achados vão de encontro com estudos de Lettry et al. (2019) que em seus estudos constatou que a maioria dos casos $(51,33 \%)$ aconteu em pessoas pardas. O fato de maior número de casos em pessoas pardas pode ter relação com a questão da grande miscieginação racial presente na população brasileira (Oliveira et al., 2020).

É possivel observar no Gráfico 4 a notificação dos casos de dengue distribuídos entre os meses do ano. Percebe-se que os meses de janeiro, fevereiro e março apresentam uma crescente na quantidade de casos notificados, a partir de abril até julho ocorre uma drastica queda na notificação, de julho em diante há uma estabilização que segue até o mês de outubro onde volta a subir novamente. 
Gráfico 4. Casos notificados de dengue segundo os meses do ano, no estado de Pernambuco, no período de 2015 a 2020.

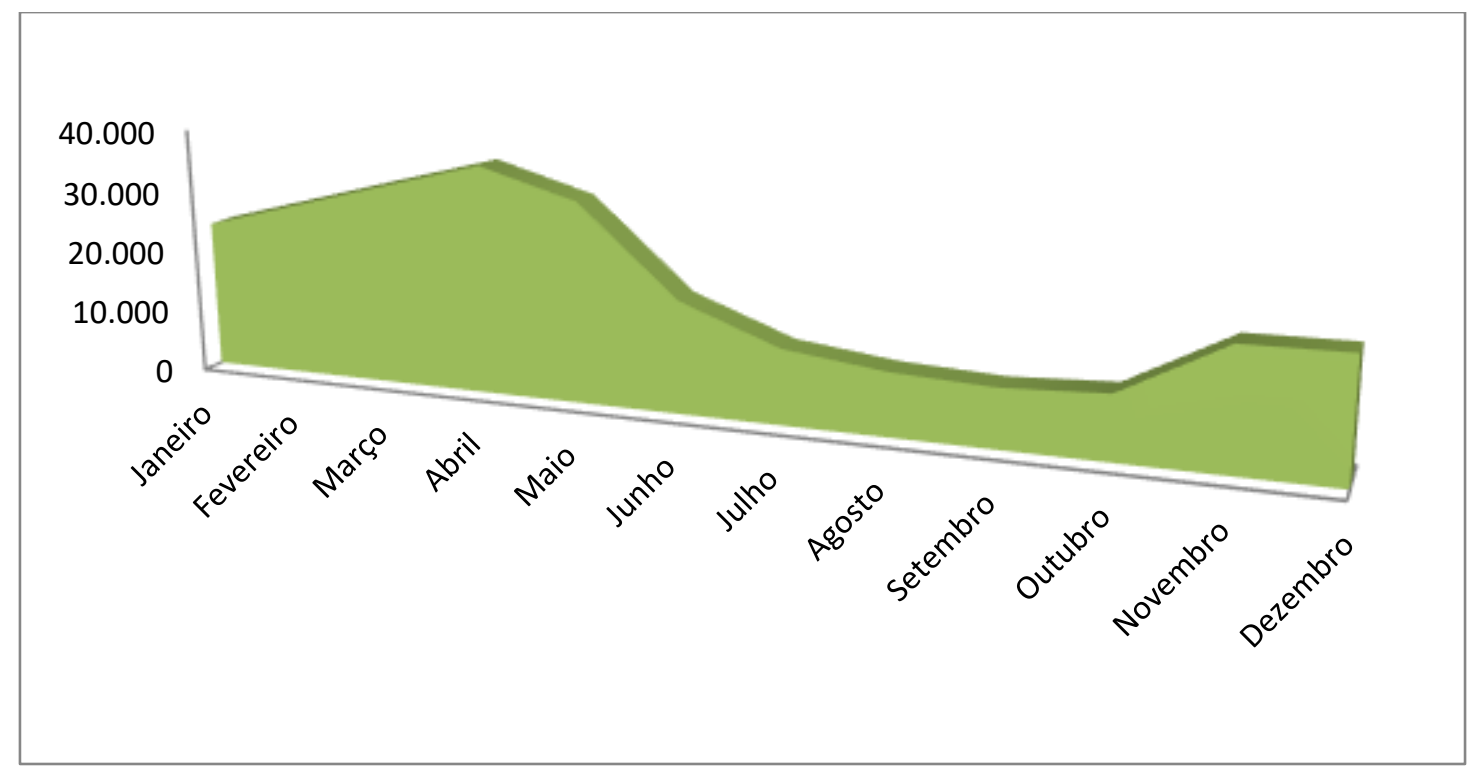

Fonte: Autores (2022). A partir de dados coletados do SINAN/DATASUS.

No Gráfico 4 é possivel perceber que a maioria dos casos aconteceram de novembro até março, período marcado principalmente pelo verão (dezembro a março), no outono (março a junho) é marcado pela drástica queda no número dos casos, no inverno (junho a setembro) e primavera (setembro a dezembro), observamos que os casos se estabiliza em pouca quantidade, sendo que no final da primavera a uma retomada de aumento dos casos, que soma com o grande aumento que acontece no verão. Achadas semelhantes foram achados nos estudos de Lucena et al. (2019) e de Rodrigues et al. (2020).

Considerandos o estudo de Câmara et al. (2009), a possível explicação para a maior ocorrência de casos no verão, pode ser o fato de que nos meses mais quentes é onde ocorre uma alta proliferação do Aedes aegypti.

Gráfico 5. Casos notificados de dengue segundo escolaridade, no estado de Pernambuco, no período de 2015 a 2020.

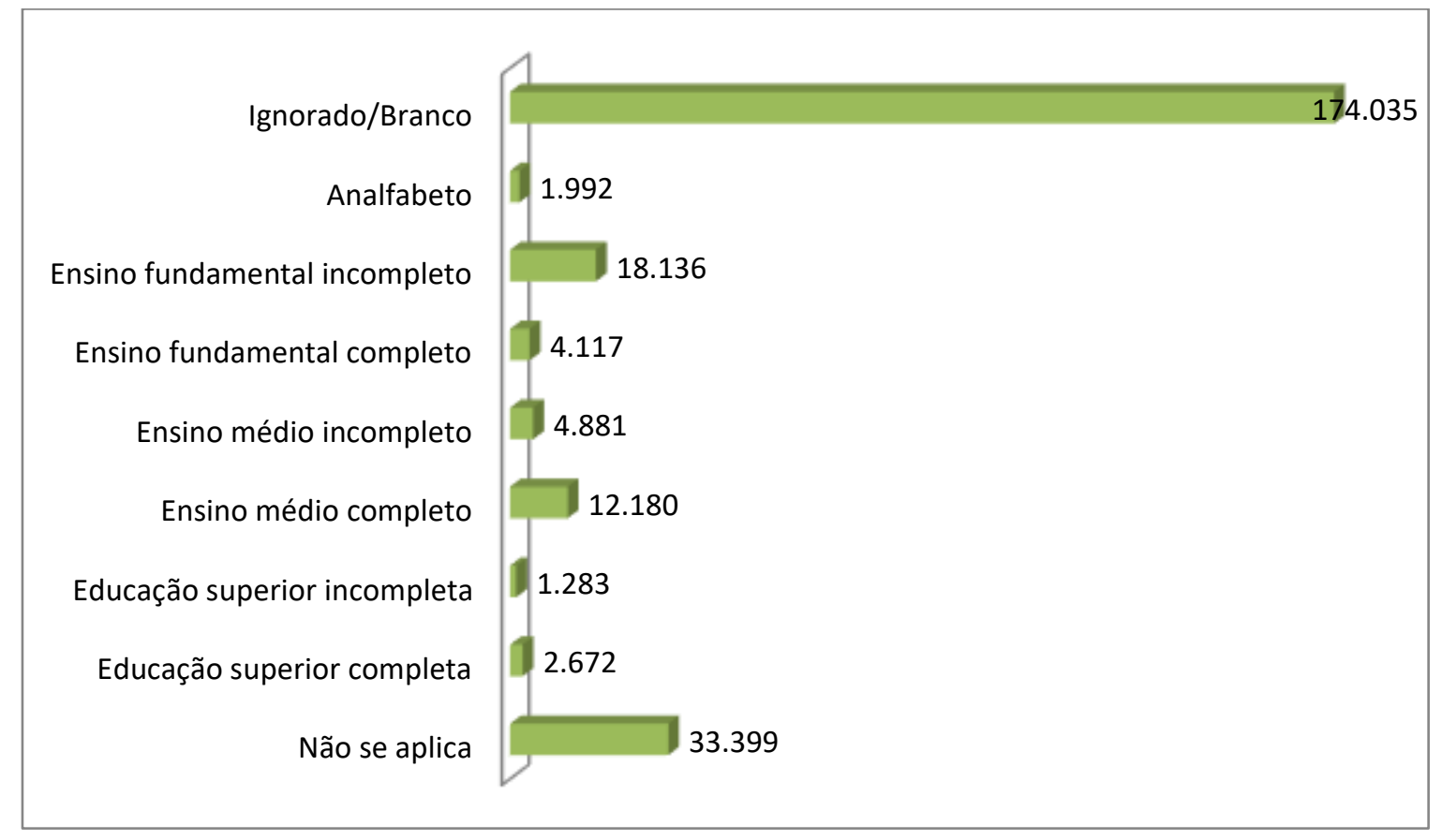

Fonte: Autores (2022). A partir de dados coletados do SINAN/DATASUS. 
No que diz a respeito a escolaridade, percebe-se que essa variável é pouco notificada sendo que a maioria dos casos foram notificados com ignorado/branco (68,9\%), essa característica se assemelha com os estudo de Silva et al. (2019) que em um estudo realizado no estado de Alagoas entre os anos de 2011 a 2015 percebeu que a maioria dos casos dessa variável eram notificados como ignorado/branco. Apesar da vulnerabilidade dos dados apresentados, aqueles que estão no ensino fundamental (incompleto ou completo) apresentam a maioria dos casos, para Cunha et al. (2015) quando mais baixo for o nível de escolaridade dos indivíduos, menor é o seu conhecimento sobre os fatores relacionados com a dengue, aumentando o risco de infecção pela dengue desses individuos.

No Gráfico 6 é mostrado como ocorreu a confirmação do caso, onde a maioria foi por criterio clínico-epidemiológico $(52,1 \%)$, sendo a confirmação laboratorial presente em apenas $8,4 \%$ dos casos.

Gráfico 6. Casos notificados de dengue segundo confirmação, no estado de Pernambuco, no período de 2015 a 2020.

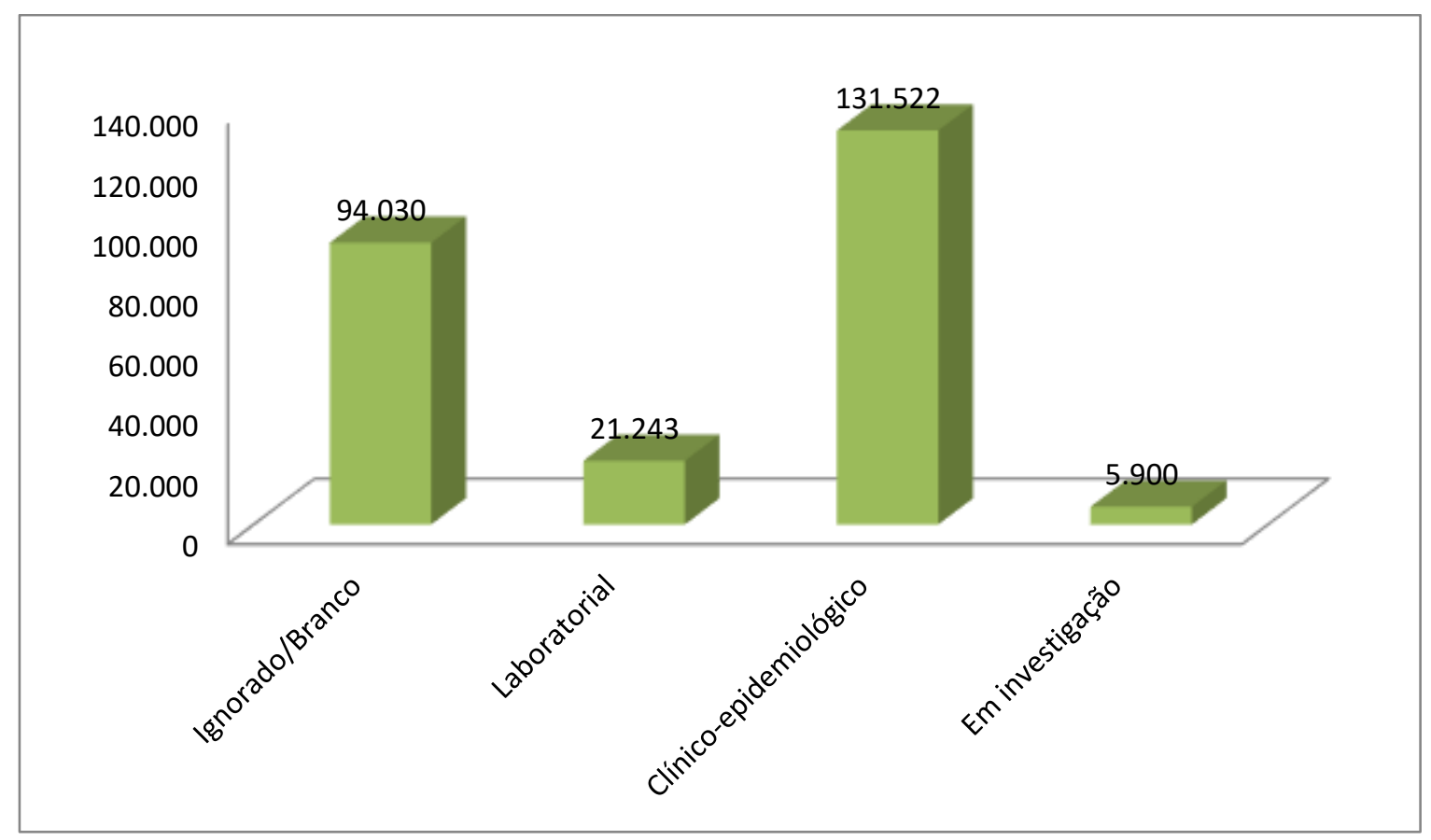

Fonte: Autores (2022). A partir de dados coletados do SINAN/DATASUS.

A confirmação do caso de dengue vai de acordo com a atual situação epidemiológica da região acerca da doença, em periodos não endêmicos a vigilância epidemiológica preconiza que todos casos suspeitos devem ser encaminhados para realização de exames laboratoriais, já em períodos endêmicos, a solicitação é realizada apenas a pacientes com quadros grave da doença que tenha alguma dúvida no diagnóstico (Assunção et al., 2014).

Em um estudo realizado em 28 pacientes que tiveram casos suspeitos de dengue, Ribeiro et al. (2008), constatou que as principais manifestações clinicas relatada pelos pacientes foram febre (100\%), cefaleia $(78,6 \%)$, artralgia e/ou mialgia $(67,9 \%)$ e vômito $(46,4 \%)$. Contudo, os exames laboratoriais são mais preciso para a confirmação dos casos de dengue, pois, segundo Viana (2016), a sintomatolozia da dengue pode ser confudida com outras arboviroses. Os principais exames laboratorias utilizados para o diagnóstico da dengue são o PT-PCR, que serve para realizar a detecção do genoma viral, o método imunoenzimático de ELISA, que detecta os anticorpos ( $\operatorname{IgM})$ em resposta da infecção viral, a identificação do antigeno NS1 na corrente sanguiena também é importante, pois esse antígeno é liberado pelas células quando são infectadas pelos DENVs (Silva et al., 2021).

Na Tabela 1 é possível observar os sorotipos dos casos de dengue, observa-se que a grande maioria foram notificados 
como ignorado/branco, os casos que tiveram essa diferenciação não somaram mais que $1 \%$ dos casos.

Tabela 1. Casos notificados de dengue segundo sorotipos, no estado de Pernambuco, no período de 2015 a 2020.

\begin{tabular}{cc}
\hline SOROTIPO & CASOS \\
\hline Ignorado/Branco & 252.306 \\
DENV1 & 361 \\
DENV2 & 12 \\
DENV3 & 10 \\
DENV4 & 6 \\
\hline TOTAL & $\mathbf{2 5 2 . 6 9 5}$ \\
\hline
\end{tabular}

Fonte: Autores (2022). A partir de dados coletados do SINAN/DATASUS.

Os quatros sorotipos (DENV 1, DENV 2, DENV 3 e DENV 4) são imunologicamente distinto, a infecção de um tipo especifico garante proteção permanente por uma segunda infecção homologa e parcialmente se for um infecção por outro sorotipo (Campos et al., 2018). Segundo Sousa et al. (2020) dos quatros tipos de vírus, o DENV 3 causa a forma mais acentuada e de maior risco ao paciente, por ser apresentar mais virulento, já o DENV 1 é o mais importante do ponto de vista epidemiologico, pois ele tem a capacidade de contaminar as pessoas de modo avassalador e rápido.

O DENV 2 é um dos sorotipos mais bem estudado, pelos metodos moléculares existente, pelo fato da sua frequente associação com epidemia e casos de dengue hemorrágica (segundo Siqueira et al., (2010) a epidemia que ocorreu no Brasil em 2008 teve predominancia desse sorotipo), apesar de não houver a associação do DENV 4 com as formas graves da doença, foi observado que os pacientes que foi infectado por esse sorotipo apresentava caractersticas raras nos aspectos clínicos da doença (Silva et al., 2014).

O Gráfico 7 mostra que a maioria dos casos 143.421 (56,76\%) evoluíram para a cura, em relação ao óbito foram notificados $307(0,03 \%)$ terminaram em óbito, no banco de dados aindam constam 307 (0,12\%) óbitos em investigação, na Tabela 2 é possivel observar que o ano de 2016 apresentou 220 (71,66\%) óbitos em investigação, o maior número do periodo estudado, sendo que apenas o ano de 2019 que não apresenta nenhum caso dessa variável.

Gráfico 7. Casos notificados de dengue segundo evolução, no estado de Pernambuco, no período de 2015 a 2020.

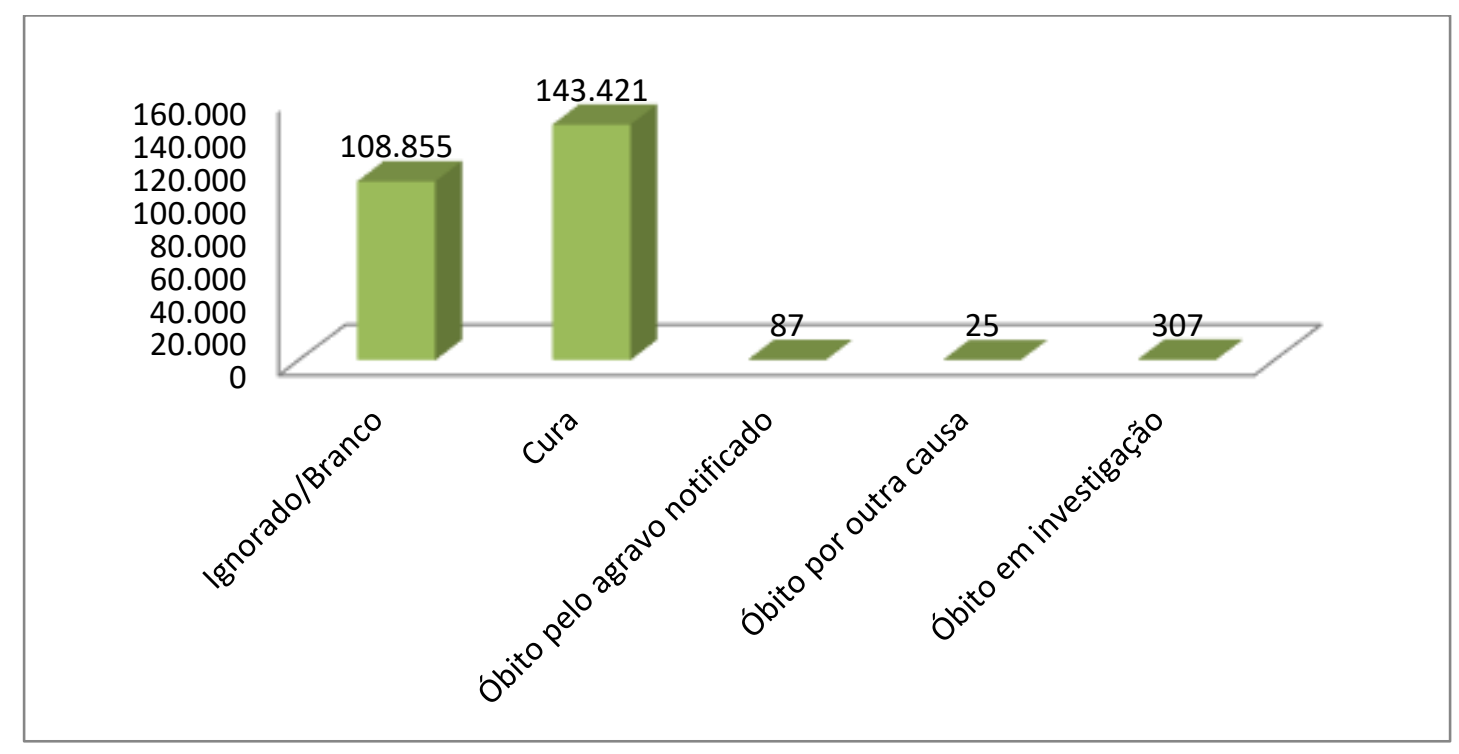

Fonte: Autores (2022). A partir de dados coletados do SINAN/DATASUS. 
Tabela 2. Casos notificados de dengue segundo óbito em investigação, no estado de Pernambuco, no período de 2015 a 2020.

\begin{tabular}{ccc}
\hline ANO & ÓBITOS EM INVESTIGAÇÃO \\
\hline 2015 & 26 \\
2016 & 220 \\
2017 & 28 \\
2018 & 14 \\
2019 & - \\
2020 & 19 \\
\hline TOTAL & $\mathbf{3 0 7}$ \\
\hline
\end{tabular}

Fonte: Autores (2022). A partir de dados coletados do SINAN/DATASUS.

Os serviços de saúde têm condições necessárias para garantir assistência a saúde adequada aos pacientes com dengue, sendo as mortes ocasionadas por ela consideradas evitáveis (Figueiroa et al., 2017). Apesar do baixo número de morte (0,03\%) notificado, na realidade pode ser bem maior, já que no Gráfico 7 observamos que existe uma grande quantidade de casos ignorado/branco (43,08\%), somando isso aos dados observados na Tabela 2, que em todo o período estudado, exceto em 2019, ainda existem óbitos em investigação, podemos constatar que existe uma falha no momento da notificação ou até mesmo uma tentativa de máscara o real número das mortes causada pela doença.

Gráfico 8. Casos notificados de dengue segundo classificação, no estado de Pernambuco, no período de 2015 a 2020.

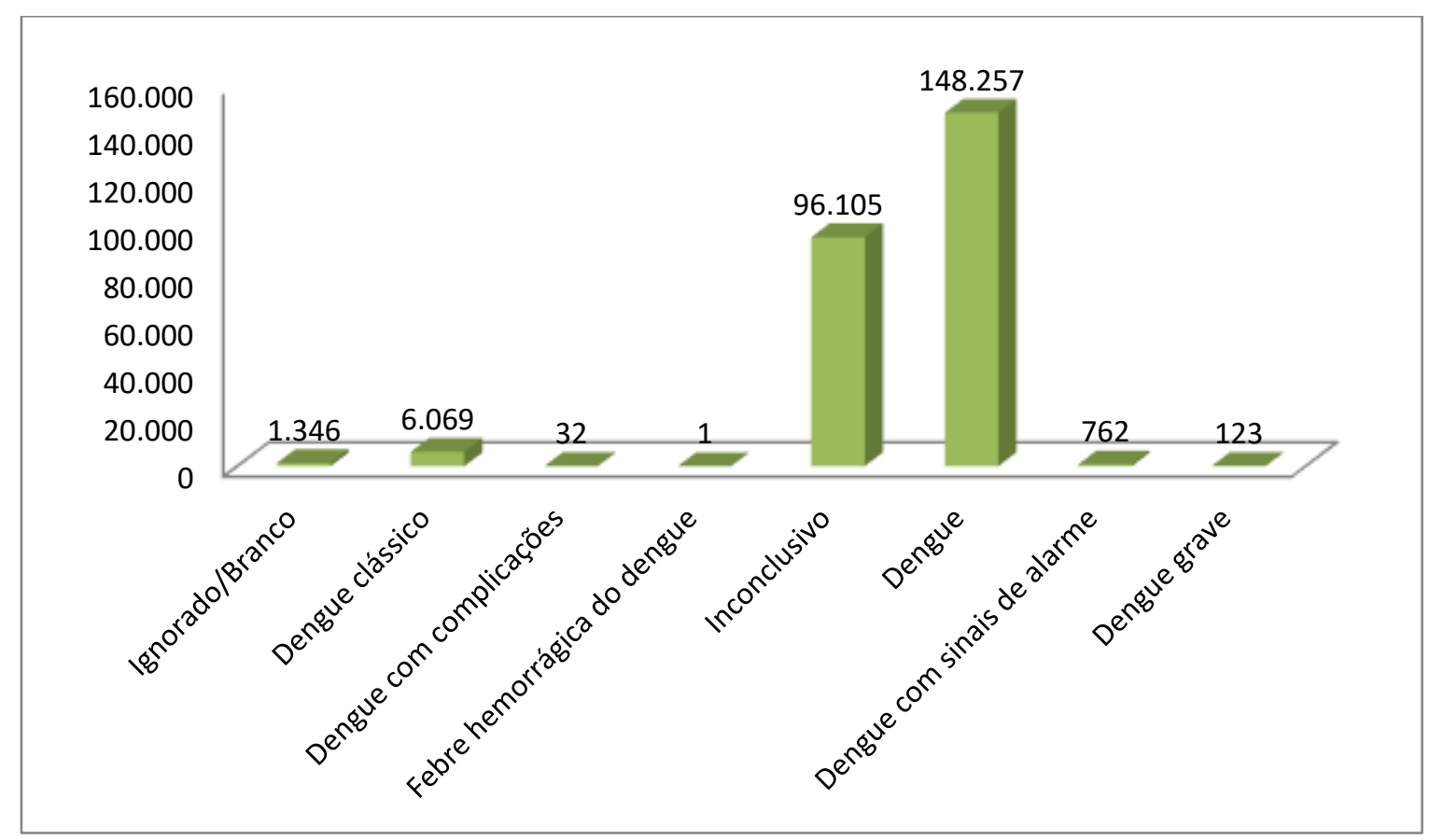

Fonte: Autores (2022). A partir de dados coletados do SINAN/DATASUS.

O Gráfico 8 mostra que a maioria dos casos $(58,67 \%)$ foram notificados como dengue, com um baixo número de dengue com sinais de alarme ou grave (0,30 e 0,05\% respectivamente), esses dados se assemelham a outros na literatura, como o de Santana et al. (2019). Contudo é possível perceber que 96.105 (38,03\%) se apresentam como inconclusivo, e com o histórico de subnotificação apresentado pelo estão, as pessoas que tiveram seu caso de dengue agravado pode ser bem maior do que apresentado nos dados.

O Gráfico 9 mostra a distribuição dos casos por faixa etária, sendo que a maioria dos casos ocorreu na faixa dos 20 a 
$39(36,08 \%)$, seguida da $40-59(21,86 \%)$ dos casos.

Gráfico 9. Casos notificados de dengue segundo faixa etária, no estado de Pernambuco, no período de 2015 a 2020

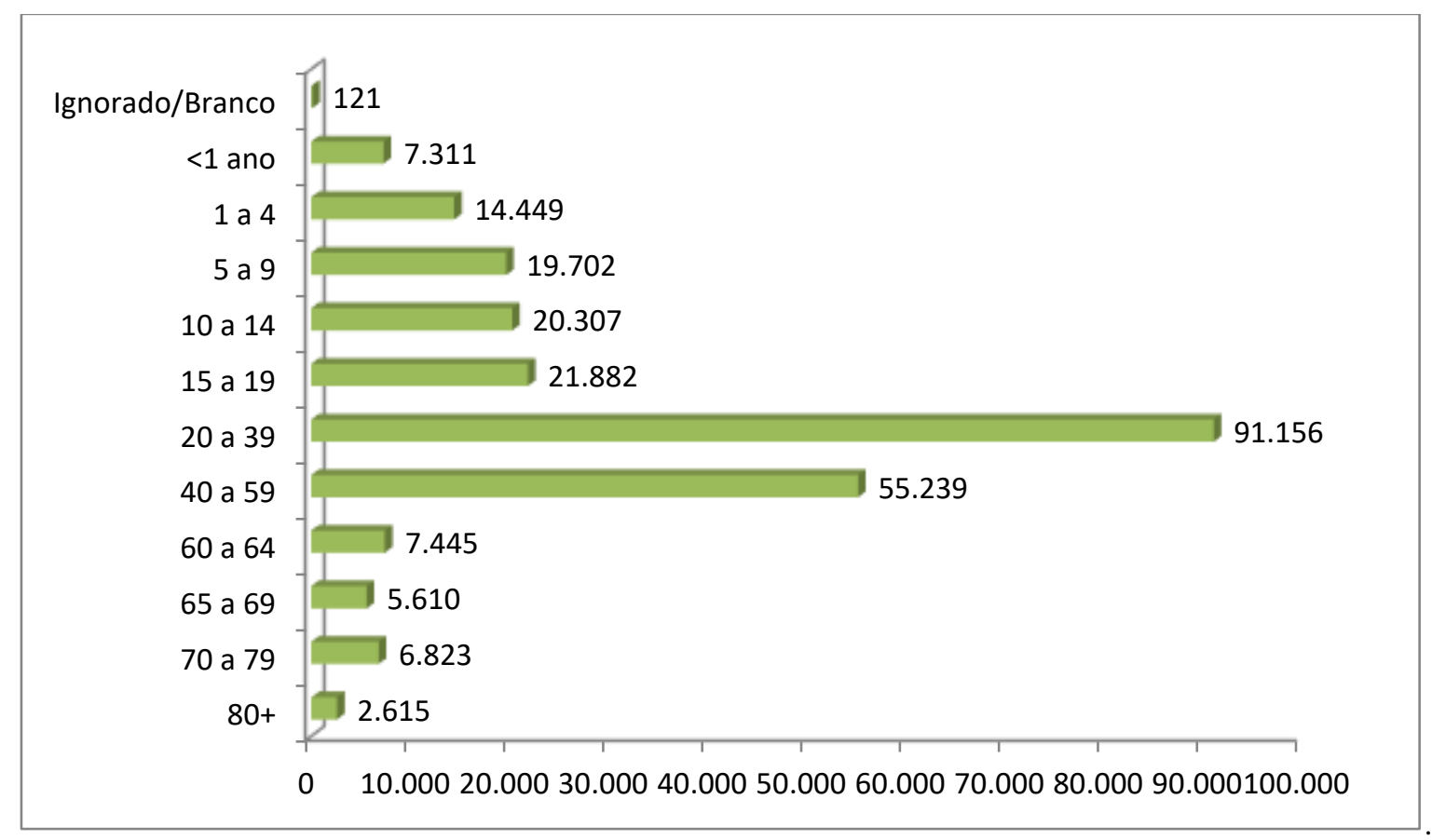

Fonte: Autores (2022). A partir de dados coletados do SINAN/DATASUS.

O Gráfico 9 mostra uma maior ocorrência de casos na faixa etária dos 20 a 39 anos, responsável por 91.156 (36,08\%) dos casos, essa informação é similar a outros achados da literatura, como o Silva et al. (2020) que constatou que 52.22\% dos casos de dengue no estado do Rio Grande do Norte foi nessa faixa etária, e o de Santos et al. (2010) que observou que no município de Anápolis a ocorrência de casos nessa faixa etária também foi predominante (46,2\%). Outros estudos realizados no estado constataram que no período de 1995-2006 mostrou uma maior quantidade de casos entre os indivíduos de 20 a 39 anos (Cordeiro et al., 2007), o que demostra uma frequência histórica de casos da doença nessa faixa etária no estado. Uma possível explicação para esse fato é que essa faixa etária faz parte da população ativa, assim, eles visitam vários ambientes diferentes, estando propenso a encontrar um Aedes aegypti em um desses ambientes e ser infectado pela dengue. Outro fato importante a ser observado é a quantidade alta de casos em crianças, essa informação vai de encontro com dados do Ministério da Saúde, que diz que a partir de 2008, a dengue foi marcada por um padrão migratório de casos graves em crianças (Brasil, 2009).

\section{Conclusão}

Podemos constatar então que a dengue ainda é um problema de saúde pública em Pernambuco, ainda se apresentando como uma epidemia local. Foi possível perceber a maioria dos casos foram em mulheres, e faixa etária de 20-39, de raça parda e com o ensino fundamental incompleto. A grande maioria dos casos foram confirmados por métodos clínicos-epidemiológico, sendo que boa parte não desenvolveu nenhuma gravidade causada pela doença e evoluindo para cura, sendo que devido ao grande fato de casos ignorado/branco não se pode compreender qual o sorotipo mais prevalente. A maior ocorrência dos casos esta relacionado com o período mais quente do ano.

O estado ainda apresenta uma grande subnotificação dos casos, sendo que em muitos casos não há uma notificação de maneira eficiente, o estado também apresenta uma certa inconsistência dos dados, que de alguma forma pode comprometer a 
formulação de políticas públicas para o seu combate.

Espera-se que os próximos estudos abrangessem principalmente a questão da subnotificação dos dados e nas deficiências apresentadas no momento da notificação pelo profissional responsável, outro ponto importante a ser abordado seria na ampliação do conhecimento sobre qual o sorotipo que está mais prevalente.

\section{Referências}

Andrioli, D. C., Busato, M. A., \& Lutinski, J. A., (2020). Características da epidemia de dengue em Pinhalzinho, Santa Catarina, 2015-2016. Epidemiol. Sérv. Saúde 29 (4) 19 Ago 2020. https://doi.org/10.5123/S1679-49742020000400007

Assunção, M. L., \& Aguiar, A. M. M. (2014). Perfil clínico-epidemiológico da dengue no município de Juscimeira - MT. Rev Epidemiol Control Infect. 2014;4(4):249-253. https://doi.org/10.17058/reci.v4i4.5605

Braga, I. A., \& Valle, D., (2007). Aedes aegypti: histórico do controle no Brasil. Epidemiologia e Serviços de Saúde 2007 ; $16(2)$ : 113 - 118. http://dx.doi.org/10.5123/S1679-49742007000200006

Brasil (2009). Ministério da Saúde. Secretaria de Vigilância em Saúde. Diretrizes Nacionais para a Prevenção e Controle de Epidemias de Dengue. https://bvsms.saude.gov.br/bvs/publicacoes/diretrizes_nacionais_prevencao_controle_dengue.pdf

Brasil (2021). Dengue. https://www.gov.br/saude/pt-br/assuntos/saude-de-a-a-z/d/dengue

Brasil (2021b). No nordeste, Alagoas registra maior maior aumento de dengue em 2021. https://www.gov.br/saude/pt-br/assuntos/noticias/20211/novembro/no-nordeste-alagoas-registra-maior-aumento-de-casos-de-dengue-em-2021

Brasil (2017). Ministério da Saúde. Secretaria de Vigilância em Saúde. Boletim epidemiológico.

Brasil (2017b). Ministério da Saúde. Secretaria de Vigilância em Saúde. Resumo executivo saúde brasil 2015/2016: Uma análise da situação de saúde e da epidemia pelo vírus Zika e por outras doenças transmitidas pelo Aedes aegypti. https://pesquisa.bvsalud.org/bvsms/resource/pt/mis-39337

Brasil (2016). Resolução $N^{O}$ 510, de 7 de Abril de 2016. https://www.in.gov.br/materia/-/asset_publisher/Kujrw0TZC2Mb/content/id/22917581

Brasil (2016b). Portaria $N^{O}$ 204, de 17 de Fevereiro de 2016. https://www.in.gov.br/materia/-/asset_publisher/Kujrw0TZC2Mb/content/id/22311994

Campos, J. S. C. B., Silva, S. W. B., Costa, T. C. P., \& Silva K. M. R., (2018). The prevalence of circulating serotypes of dengue virus in the state of piauí in the year 2011 to 2013. ReonFacema. 2018 4(Esp):937-951. file:///C:/Users/Carlos/Downloads/380-1387-1-PB.pdf

Câmara, F. P., Gomes, A. F., Santos, G. T., \& Câmara, D. C. P., (2009). Climas e epidemias de dengue no Estado de Rio de Janeiro. Rev. Soc. Bras. Med. Trop. 42 (2). https://doi.org/10.1590/S0037-86822009000200008

Cavalcanti, L. P. G., Barreto, F. K. A., Oliveira, R. M. A. B., Canuto, I. F. P., Lima, A. A. B., Lima, J. W. O., Escóssia, K. N. F., Martins, V. E. P., Alencar, C. H., Perdigão, A. C. B., Lima, D. M., Ramalho, I. L. C., \& Araújo, F. M. C., (2018). Trinta anos de dengue no Ceará: história, contribuições para ciência e desafios no cenário atual com tripla circulação de arbovírus. J. Health Biol Sci. 2018; 6(1):65-82. http://dx.doi.org/10.12662/2317-3076jhbs.v6i1.1415.p6582.2018

Cunha, T. H. \& Hamad, G. B. N. Z. Condições ambientais como fator de risco na prevalência da dengue. http://www.editorarealize.com.br/revistas/enect/trabalhos/Comunicacao_249_2.pdf

Cordeiro, M. T., (2008). Evolução da dengue no estado de Pernambuco, 1987-2006: epidemiologia e caracterização molecular dos sorotipos circulantes. 2008. 226 f. Tese (Doutorado em Saúde Pública) - Fundação Oswaldo Cruz, Centro de Pesquisas Aggeu Magalhães, Recife, 2008. https://www.arca.fiocruz.br/handle/icict/3890

Cordeiro, M. T., Schatzmayr, H. G., Nogueira, R. M. R., Oliveira, V. X., Melo, W. R., \& Carvalho, E. F., (2007). Dengue e febre hemorrágica do dengue no Estado de Pernambuco, 1995-2006. Rev. Soc. Bras. Med. Trop. 40 (6). https://doi.org/10.1590/S0037-86822007000600001

Dias, L. B. A., Almeida, S. C. L., Haes, T. M., Mota, L. M., \& Roriz-Filho, J. S., (2010). Dengue: Transmissão, aspectos clínicos, diagnóstico e tratamento. Medicina (Ribeirão Preto) 43(2): 143-52. https://doi.org/10.11606/issn.2176-7262.v43i2p143-152

Duarte, H. H. P., \& França, E. B. (2006). Qualidade dos dados da vigilância epidemiológica da dengue em Belo Horizonte, MG. Revista de Saúde Pública 40(1): 134-142. https://doi.org/10.1590/S0034-89102006000100021

Figueiroa, M. N., Menezes, M. L. N. M., Barbosa, A. M., Silva, I. T. C., Monteiro, E. M. L. M., \& Santana, A. D. L., (2017). Prevalence of Arbovirus Infections among Pregnant Women at a Maternity Hospital School. International Archives Of Medicine, 10. https://doi.org/10.3823/2412

Guedes, D. A. M. O., \& Rocha, B. A. M. (2019). Perfil epidemiológico dos casos de dengue notificados em Ceres-Goiás, de 2014 a 2015. Revista de Epidemiologia e Controle de Infecção, Santa Cruz do Sul, v. 9, n. 2, maio 2019. https://doi.org/10.17058/reci.v9i2.11396

Hochman, B., Nahas, F. X., Filho, R. S. O., \& Ferreira, L. M., (2005). Desenhos de pesquisas. Acta cirúrgica brasileira - 20 (2). https://doi.org/10.1590/S0102-86502005000800002

Instituo Brasileiro de Geografia e Estatísticas (2021). Brasil/Pernambuco. https://cidades.ibge.gov.br/brasil/pe/panorama 
Lettry, T. C. R. N., Tobias, G. C., \& Teixeira, C. C., (2021). Perfil epidemiológico de dengue em senador canedo - Goiás, Brasil. Uningá Journal, 58, eUJ3722. http://34.233.57.254/index.php/uninga/article/view/3722

Lucena, L. C., Souto, A. A., Lucena, L. C., \& Marques, T. N. (2019). avaliação do perfil epidemiológico dos casos de dengue no município de porto nacional, Tocantins. Revista de Patologia do Tocantins, 6(1), 18-23. https://doi.org/10.20873/uft.2446-6492.2019v6n1p18

Nascimento, I. D. S., Pastor, A. F., Lopes, T. R. R., Farias, P. C. S., Gonçales, J. P., Carmo, R. F., Durães-Carvalho, R., Silva, C. S., \& Silva Júnior, J. V. P., (2020). Retrospective cross-sectional observational study on the epidemiological profile of dengue cases in Pernambuco state, Brazil, between 2015 and 2017. BMC Public Health (2020) 20:923. https://doi.org/10.1186/s12889-020-09047-z

Oliveira, E. H., Rodrigues, F. R., Coêlho, M. B., Verde, R. M. C. L., \& Sousa, F. C. A. (2020). Análise epidemiológica dos casos de dengue no Estado do Maranhão, Brasil. Research, Society and Development, 9(4), e78942491. https://doi.org/10.33448/rsd-v9i4.2491

Oliveira, E. H., Soares, J. dos S., Acha, B. T., Verde, R. M. C., Soares, L. F., \& Lima, H. R. (2019). Impacto epidemiológico da dengue no estado da Paraíba, Brasil. Research, Society and Development, 8(12), e488121947. https://doi.org/10.33448/rsd-v8i12.1947

Ribeiro, P. C., Sousa, D. C., \& Araújo, T. M. E., (2008). Perfil clínico-epidemiológico dos casos suspeitos de Dengue em um bairro da zona sul de Teresina, PI, Brasil. Rev Bras Enferm, Brasília 2008 Rev Bras Enferm, Brasília 2008 mar-abr; 61(2): 227-32. https://doi.org/10.1590/S0034-71672008000200013

Rocha, M. S., Bartholomay, P., Cavalcanate, M. V., Medeiros, F. C., Codenotti, S. B., Pelissari, D. M., Andrade, K. B., Silva, G. D. M., Sanchez, D. A., \& Pinheiro, R. S., (2020). Sistema de Informação de Agravos de Notificação (Sinan): principais característica da notificação da análise de dados relacionados à tuberculose. Epidemiol. Serv. Saude, Brasília, 29(1):e2019017, 2020. https://doi.org/10.5123/S1679-49742020000100009

Rodrigues, A. E. P., Campos, J. C. B., Oliveira, I. D., Batista, K. C., Okabaiashi, D. C. V., Ribeiro, S. M. G., Reis Júnior, O. M., \& Bitencourt, E. L., (2020). Perfil epidemiológico da dengue em palmas de 2015 a 2017. Revista de Patologia do Tocantins 2020; 7(3). https://sistemas.uft.edu.br/periodicos/index.php/patologia/article/view/10493/17615

Rio Grande do Sul (2021). Aedes aegypti - dengue, zika e chikungunya. https://saude.rs.gov.br/aedes

Santana, V. T. P., \& Duarte, P. M., (2019). Perfil epidemiológico dos casos de dengue registrados no município de Primavera do Leste-Mt entre o período de 2002 a 2012. Braz. J. of Develop., Curitiba, v. 5, n. 11, p. 27508-27518, nov. 2019. https://www.brazilianjournals.com/index.php/BRJD/article/view/4982

Santo, A. C. G. E., Fernando, V. C. N., \& Bezerra, A. F. B., (2012). Despesa pública municipal com saúde em Pernambuco, Brasil, de 2000 a 2007. Ciência e saúde coletiva 17 (4), Abril 2012. https://doi.org/10.1590/S1413-81232012000400009

Santos, C. H., Sousa, F. Y. de, Lima, L. R. de, \& Stival, M. M. (2010). Perfil Epidemiológico do Dengue em Anápolis-GO, 2001 - 2007. Revista De Patologia Tropical / Journal of Tropical Pathology, 38(4), 249-260. https://doi.org/10.5216/rpt.v38i4.8588

Santos, L. C. G. B., Silva, N. L., Vasconcelos, B. M., Ferreira, K. B. A. N., Ferreira, L. C., Santos, R. F. E. P., Silva, L. S. M., \& Pontes, A. N., (2019). Perfil epidemiológico do estado de Alagoas relacionado com á arbovirose dengue. Braz. J. Hea. Rev., Curitiba, v. 2, n. 3, p. 1604-1608, mar./apr. 2019. https://www.brazilianjournals.com/index.php/BJHR/article/view/1406

Santos, L. K. F., Pessôa, V. M. L., Santos, T. S. dos, Bragagnollo, G. R., Machado, G. P., Munõz, S. I. S., Bragagnollo, S. R., Guimarães, M. N., \& Carvalho, L. W. T., (2019). Perfil epidemiológico da dengue em um estado do nordeste brasileiro, 2011 a 2015. Revista Eletrônica Acervo Saúde, 11(10), e423. https://doi.org/10.25248/reas.e423.2019

Scandar, S. A. S., Vieira, P., Cardoso Junior, R. P., Silva, R. A. D., Papa, M., \& Sallum, M. A. M. (2010). Dengue em São José do Rio Preto, Estado de São Paulo, Brasil, 2005: fatores entomológicos, ambientais e socioeconômicos. BEPA. Boletim Epidemiológico Paulista (Online), 7(81), 04-16. http://periodicos.ses.sp.bvs.br/scielo.php?script=sci_arttex-t\&pid=S1806-42722010000900001\&lng=pt

Silva, R. E. da ., Silva , A. C. A. da ., Araújo, D. D. ., Alves, N. S., Oliveira, A. A. de ., Santos , I. L., Mendes , M. S. S., \& Ramos, J. C. F. . (2021). Análise da realização dos exames laboratoriais no diagnóstico da Dengue no Piauí. Research, Society and Development, 10(12), e531101220776. https://doi.org/10.33448/rsd-v10i12.20776

Silva, G. M., \& Andrade A. M. M. S., (2014). Avaliação do perfil epidemiológico da dengue no município de Paripiranga, Bahia, Brasil. Scientia Plena 10, 097501(2014). https://scientiaplena.org.br/sp/article/view/1894

Silva, I. K. M., Medeiros, E. R., Silva, B. C. O., Paiva, M. D. B., Barreto, V. P., \& Feijão, A. R., (2020). Série histórica dos casos de dengue no estado do Rio Grande do Norte, Brasil. Revista Uruguaya de Enfermería, 2020; 15(2): 1-13. https://pesquisa.bvsalud.org/portal/resource/pt/biblio-1283526?src=similardocs

Souza, A. I. S., Gomes Junior, A. L.., Abreu, J. L. S., Sampaio, J. P. S., Sousa, L. G., \& Chaves, T. V. S. (2020). Casos notificados de dengue no Estado do Piauí entre os anos de 2015 a 2019. Research, Society and Development, 9(11), e59691110231. http://dx.doi.org/10.33448/rsd-v9i11.10231

Teixeira, M. G., Siqueira Junior, J. B., Ferreira, J. L. C., Bricks, L., \& Join, G., (2013). Epidemiological trends of dengue disease in Brazil (2000-2010): A systematic literature search and analysis. Literature Search and Analysis. PLoS Negl Trop Dis 7(12): e2520. https://doi.org/10.1371/journal.pntd.0002520

Viana, J. A., (2016). Análise da correlação entre arboviroses epidêmicas no Brasil (dengue, chikungunya e zika) e a síndrome de Guillain-Barré: revisão sistemática de literatura. https://repositorio.ufba.br/handle/ri/21315

Zara, A. L. S. A., Santos, S. M., Fernandes-Oliveira, E. S., Carvalho, R. G. \& Coelho, G. E., (2016). Estratégias de controle do Aedes aegypti:uma revisão. Epidemiologia e Serviços de Saúde, 25(2), 391-404. http://dx.doi.org/10.5123/S1679-49742016000200017 